\title{
A Study on Optimization of Compact High-voltage Generator Based on Magnetic-core Tesla Transformer
}

\author{
Young-Kyung Jeong*, Dong-Gi Youn** and Moon-Qee Lee ${ }^{\dagger}$
}

\begin{abstract}
This paper presents a compact and portable high-voltage generator based on magneticcore Tesla transformer for driving an UWB high power electromagnetic source. In order to optimize the performance of the high-voltage generator, a novel open-loop cylindrical magnetic-core adopting the quad-division lamination structure is proposed and manufactured. The designed high-voltage generator using the proposed magnetic core has a battery-powered operation and compact size of 280 $\mathrm{mm} \times 150 \mathrm{~mm}$ in length and diameter, respectively. The high-voltage generator can produce a voltage pulse waveform with peak amplitude of $450 \mathrm{kV}$, a rise time of $1.5 \mathrm{~ns}$, and pulse duration of $2.5 \mathrm{~ns}$ at the $800 \mathrm{~V}$ input voltage.
\end{abstract}

Keywords: High power electromagnetics, Ultra-wideband source, High-voltage generator, Tesla transformer, Pulse generator.

\section{Introduction}

Recently, the vulnerability of electronic devices to high power electromagnetic (HPEM) threats has been studied widely [1]. Ultra-wideband (UWB) HPEM source capable of producing output power in the giga watts range allows real investigation of susceptibility of electronic devices as well as their protection and hardening against HPEM threats [2]. In the future, UWB HPEM source tends to be higher pulse repetition rate, compact and portable size for the efficient testing in a variety of conditions.

Marx generators are usually applied for the generation of high-voltage pulse power to drive the UWB HPEM source. However, besides huge and complex, it is difficult to operate under high repetition rate [3]. In order to circumvent these problems, a magnetic-core Tesla transformer with compact structure and high coupling coefficient can be an alterative of Marx generator. The magnetic-core Tesla transformer, which is Tesla transformer with open-loop cylindrical magnetic-core, was first proposed by Russian scientists [4]. The performance of the transformer is dependent on the magnetic-core properties. Particularly, as the transformer become smaller, its performance degrades significantly due to the core loss and magnetic saturation which are proportional to the operation frequency. Hence, the use of low loss magnetic-core is required to achieve good performance of the transformer.

So far high-voltage pulse power generator based on the magnetic-core Tesla transformer has been widely developed. The most commonly known systems are SINUS and

$\dagger$ Corresponding Author: Dept. of Electrical and Computer Engineering, University of Seoul, Korea. (mqlee@uos.ac.kr)

* Replex Co., Ltd., Korea and Dept. of Electrical and Computer Engineering, University of Seoul, Korea. (ykj@replex.co.kr)

** Replex Co., Ltd., Korea. (admin@replex.co.kr)

Received: September 5, 2013; Accepted: January 9, 2014
RADAN series [5]. However, there have been no research results for the magnetic-core structure applicable to the Tesla transformer) In this paper, a novel magnetic-core adopting the quad-division lamination structure is proposed. Also, the high-voltage generator using the proposed magneticcore is designed and tested.

\section{Parameters Analysis of magnetic-core Tesla Transformer [6, 7]}

Magnetic-core Tesla transformer consists of a primary circuit, a secondary circuit, and the magnetic-cores. The primary circuit is formed by a series of a primary winding and a capacitor. The secondary circuit is also formed by a series of the secondary winding and a coaxial-type capacitor. The magnetic-cores enhance the magnetic coupling between the primary and the secondary circuits. At the same time, it plays a role of the inner and the outer conductors of the coaxial-type secondary capacitor.

The structure and magnetic field distribution of the magnetic-core Tesla transformer is shown in Fig. 1. Here $r_{i}$ and $r_{o}$ represent the outer radius of the inner magneticcore and the inner radius of the outer magnetic-core, respectively. The effective cross-sectional area of the inner and the outer magnetic-core are marked as $A_{e 1}$ and $A_{e 2}$, respectively. The $l_{T}$ is the length of the magnetic-core with relative permeability $\mu_{r}$. The $l_{K}$ is the overlap length of the primary and the secondary winding. The $N_{1}$ and $N_{2}$ represent the turn numbers of the primary and the secondary windings, respectively.

For simplicity we assume that the axial magnetic field in the magnetic-cores is uniform and the influence of eddy current is ignored. The magnetic fields of the outer and the inner magnetic-cores are represented by $H_{11}$ and $H_{12}$, 


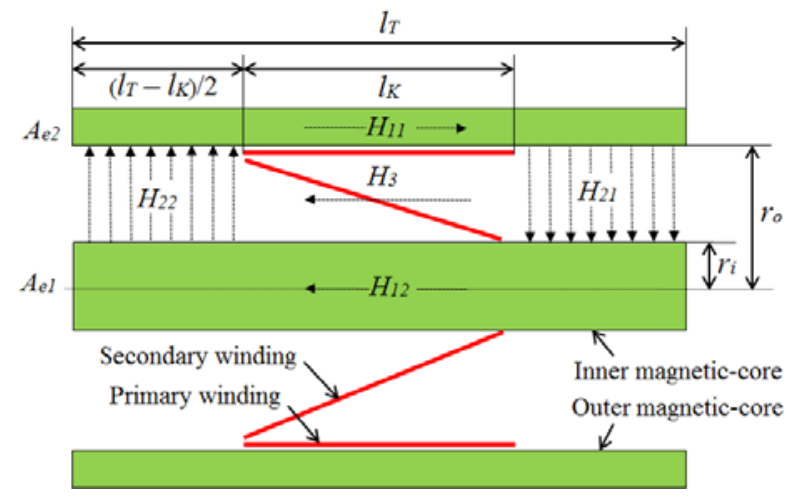

Fig. 1. Structure and magnetic field of the Tesla transformer

respectively. The fields in the spacing between the magneticcores include a radial and an axial component, which are $H_{21}, H_{22}$, and $H_{3}$, respectively.

Since the main magnetic flux of the transformer is determined by a radial component, the axial component $\mathrm{H}_{3}$ is ignored in the analysis. Assuming the primary winding current $I_{l}$ is uniformly distributed in the primary winding which is located at middle of the magnetic-core, boundary conditions are the same at both ends of the magnetic-core. Consequently, the transformer has a symmetrical magnetic field distribution. Thus, if the effective cross-sectional area of inner and outer magnetic-cores are equal, that is $A_{e 1}=A_{e 2}=A_{e}$, the magnetic field in the magnetic-cores $H_{1}$ and the magnetic field in the spacing between the magnetic-cores $H_{2}$ can be defined as follows, respectively

$$
\begin{aligned}
& H_{1} \equiv H_{11}=H_{12} \\
& H_{2} \equiv H_{21}=H_{22}
\end{aligned}
$$

According to the flux conservation of inner and outer magnetic-cores, $\Phi=A_{e} H_{1}=A_{e} H_{2}(r), \quad r_{i} \leq r \leq r_{o}$, the relationship between $H_{1}$ and $H_{2}$ is given by

$$
\mu_{r} A_{e} H_{1}=r H_{2}(r) \pi\left(l_{T}-l_{K}\right)
$$

From Ampere's circuital law, the relationship between current and magnetic field is as follows

$$
H_{1} l_{T}+H_{2} r_{i} \ln \beta=\frac{1}{2} N_{1} I_{1}
$$

where $\beta=r_{o} / r_{i}$.

Relationship of the storage energy in the magnetic-cores $W_{1}$ and the storage energy in the spacing between the magnetic-cores $W_{2}$, substituting Eq. (3), gives the following:

$$
\frac{W_{1}}{W_{2}}=\frac{\frac{1}{2} \int \mu_{r} H_{1}^{2} d V}{\frac{1}{2} \int \mu_{0} H_{2}^{2} d V}=\frac{\pi l_{T}\left(l_{T}-l_{K}\right)}{\mu_{r} A_{e} \ln \beta}
$$

where $\mu_{0}$ is the permeability of free space. Magnetic field energy is mainly concentrated in the spacing between magnetic-cores because $\mu_{r}>>\pi l_{T}\left(l_{T}-l_{K}\right) / A_{e} \ln \beta$. Therefore, the first term of Eq. (4) can be neglected, and then magnetization inductance $L_{\mu}$ is defined as follows

$$
L_{\mu}=\frac{1}{I^{2}} \int B \bullet H d V=\frac{\pi}{2} N_{I}^{2} \frac{l_{T}-l_{K}}{\ln \beta}
$$

Assuming that $H_{P}$ is the magnetic field produced by the primary winding current $I_{1}$ and $H_{S}$ is the reverse magnetic field produced by the secondary winding current $I_{2}$, leakage inductance $L_{s}$ is represented by

$$
L_{s}=\frac{1}{I_{I}^{2}} \int\left(H_{P}-H_{S}\right)^{2} d V
$$

The method for calculating the magnetic field produced by the secondary winding is the same as that for the primary windings described above, therefore, leakage inductance $L_{s}$ is defined as follows

$$
L_{s}=\frac{\pi}{3} N_{1}^{2} \frac{r_{o}^{2}}{l_{K}} \frac{(\beta-1)(2 \beta-1)}{\beta^{2}}
$$

If the coupling coefficient $K$ has a value close to 1 , according to the Eqs. (6) and (8), $K$ is defined by

$$
K=\sqrt{1-\frac{L_{s}}{L_{\mu}}}=\left[1-\frac{2}{3} \frac{r_{o}^{2}}{l_{K}\left(l_{T}-l_{K}\right)} F(\beta) \ln \beta\right]^{\frac{1}{2}}
$$

where $F(\beta)=(\beta-1)(2 \beta-1) / \beta^{2}$.

Assume the leakage inductance of the primary winding $L_{s 1}$ equals that of the secondary winding $L_{s 2}$, that is $L_{s 1}=L_{s 2}=L_{s}$. So the inductances of the primary and the secondary windings, $L_{1}$ and $L_{2}$, are calculated as follow

$$
\begin{gathered}
L_{1}=L_{\mu}+L_{s} \\
L_{2}=\left(N_{2} / N_{1}\right)^{2} L_{\mu}
\end{gathered}
$$

\section{Design and Fabrication of the Magnetic-core}

\subsection{Design of the magnetic-core structure factor}

The coupling coefficient of the transformer, $K$, as shown in Eq. (9), is determined by a geometric factor such as the length and the radius of the magnetic-core. Optimization of the magnetic-core geometric structure is required to maximize $K$.

The length of magnetic-core is designed by the required pulse length, which is given by 


$$
l_{T}=\frac{\tau c}{2 \sqrt{\varepsilon_{r}}}
$$

where $\tau$ is pulse width, $\varepsilon_{r}$ is the dielectric constant of the insulating material used as dielectric in the secondary capacitor, and $c$ is the light velocity in free space.

The radius of the magnetic-cores is designed in consideration of the dielectric strength of the secondary capacitor. If $\beta$ is satisfied with the following equation, the withstand voltage of the secondary capacitor has the maximum value.

$$
\beta=\frac{r_{o}}{r_{i}}=e \approx 2.72
$$

As shown in Fig. 2 for Eq. (9), when $l_{T}$ and $\beta(=e)$ have a constant value, $K$ can be maximized in a condition of $l_{K}=l_{T} / 2$. From Fig. 2, a significant relationship between the coupling coefficient $K$ and the aspect ratio $l_{T} / r_{o}$ can be inferred; $K$ becomes larger in proportion to the aspect ratio. So, choosing the length of the magneticcore properly can lead to a larger $K$. However, larger aspect ratio is also limited by the engineering implementation. In this paper, an aspect ratio of 5:1 is achieved in consideration of the transformer size and fabrication feasibility.

The effective cross-sectional area of magnetic-core must be designed in consideration of the magnetic saturation. To avoid the saturation of the magnetic material, the effective cross-sectional area of magnetic-core should be determined by the condition which the maximum deviation of magnetic induction in magnetic-core is less than the saturation magnetic induction of the material. The effective cross-sectional area of magnetic-core $A_{e}$ can be described in the expression [8].

$$
A_{e} \geq \frac{V_{\max } T_{c h}}{2 \Delta B_{\max } N_{2}}
$$

where $\Delta B_{\max }$ is the maximum deviation of magnetic

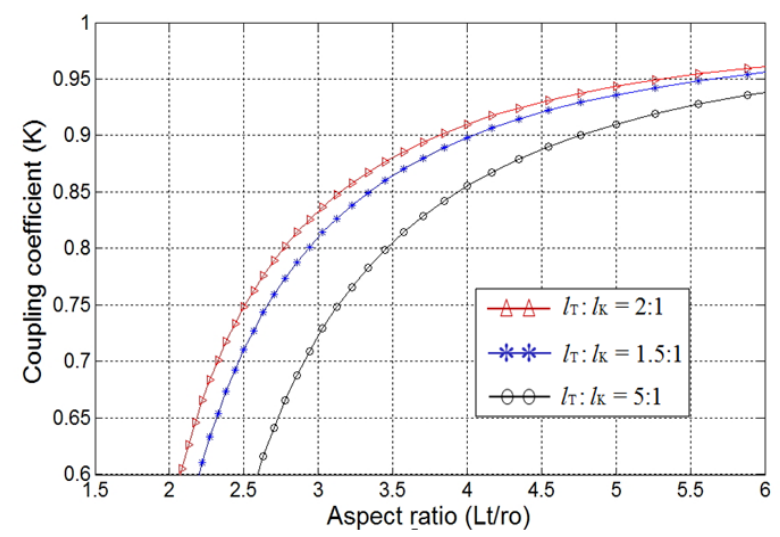

Fig. 2. Relationships between structure factor of the magnetic-core and coupling coefficient. induction in magnetic-core, $V_{\max }$ is the maximum charging voltage of secondary capacitor, and $T_{c h}$ is the charging time.

\subsection{Manufacture of the proposed magnetic-core}

The performance of magnetic-core is determined by magnetic material and structure. A highly conductive magnetic material should be used because magnetic-core is act as the conductor of secondary capacitor of the transformer. Also, the loss of magnetic-core can be reduced by structure of magnetic-core with laminated thin magnetic sheets.

Considering the above-mentioned issues, we proposed a novel open-loop cylindrical magnetic-core adopting the quad-division lamination structure. As shown in Fig. 3, the proposed magnetic-core is composed of the lamination body, ultra-thin magnetic sheets, and the cross-shaped frame. Lamination body is formed by stacked lamination of the rectangle magnetic sheets in the same size. This lamination method makes it easy to fabricate the lamination body and maximize the stacking factor. Lamination area is divided into quad-area by the cross-shaped frame which is used to support the lamination body. The surface of the crossshaped frame is curved identically to the outside curvature of the magnetic-core. Lamination bodies are arranged so that their lamination directions are orthogonal each other in the adjacent areas. These structural features are effective to reduce material damage and stress that occur during the cutting work for forming into cylindrical shape.

The photograph of the proposed magnetic-core is shown in Fig. 4. Material of the magnetic-core adopts nonoriented silicon steel with a thickness of $0.1 \mathrm{~mm}$ and saturation flux density of 1.8 Tesla.

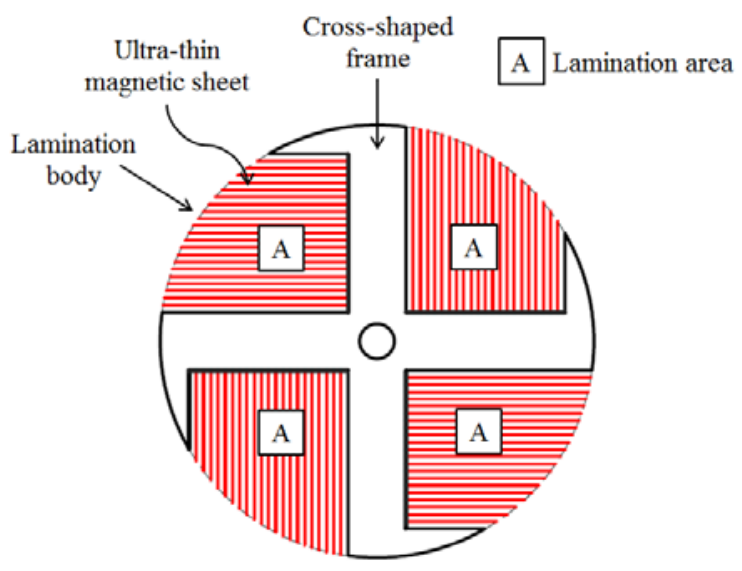

Fig. 3. Cross section of the proposed magnetic-core
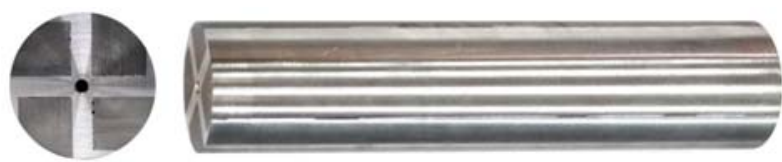

Fig. 4. Photograph of the proposed magnetic-core 


\section{Development of High-voltage generator Based on Magnetic-core TESLA Transformer.}

\subsection{Design and construction of high-voltage generator}

Electrical parameters for designing the Tesla transformer are shown in Table 1 . Where $C_{1}$ and $C_{2}$ are capacitances of the primary and the secondary capacitors, respectively. $R_{l}$ and $R_{2}$ are resistances of the primary winding and the secondary windings, respectively. $R_{k 1}$ and $L_{k 1}$ are a stray inductance and a stray resistance of the primary circuit, respectively.

A high-voltage generator based on Tesla transformer with the proposed magnetic-core is designed and constructed. The prime electric energy of the generator, as shown in Fig. 5 , is supplied by a rechargeable battery (DC $48 \mathrm{~V}, 7 \mathrm{Ah}$ ), and the primary capacitor $C_{1}$ is charged to the initial voltage $800 \mathrm{~V}$ through the full-bridge DC-DC converter and pulse charger. The primary winding $L_{1}$ is made of a copper strip with a thickness of $0.5 \mathrm{~mm}$, the conical

Table 1. Electrical parameters of the Tesla transformer

\begin{tabular}{c|c|c|c|c}
\hline Parameters & $L_{l}$ & $L_{2}$ & $C_{1}$ & $C_{2}$ \\
\hline Values & $237 \mathrm{nH}$ & $133 \mathrm{mH}$ & $25 \mathrm{uF}$ & $40 \mathrm{pF}$ \\
\hline Parameters & $R_{l}$ & $R_{2}$ & $R_{k l}$ & $L_{k l}$ \\
\hline Values & $0.1 \mathrm{~m} \Omega$ & $200 \Omega$ & $17 \mathrm{~m} \Omega$ & $40 \mathrm{nH}$ \\
\hline Parameters & $L_{s}$ & $L_{\mu}$ & $K$ & \\
\hline Values & $19 \mathrm{nH}$ & $227 \mathrm{nH}$ & 0.93 & \\
\hline
\end{tabular}

secondary winding $L_{2}$ is made of 700 turns of a 0.18 mm-diameter copper wire.

Components of the generator, as shown in Fig. 6, are housed in the pressure vessel. Insulation oil is filled in the Tesla transformer, which is pressurized through an oil conservator in order to raise their electric insulation level. The spark-gap switch is filled with insulation gas. Two capacitive voltage dividers CD-1, CD-2 are used to measure the charging voltage of the secondary capacitor $C_{2}$ and the output voltage of the high-voltage generator, respectively. A capacitive voltage divider is made up of a dielectric film of $0.15 \mathrm{~mm}$ thickness covered with copperclad in both sides. Their self-capacitances are $765 \mathrm{pF}$ and $479 \mathrm{pF}$, respectively. Their division ratios are 1:19300 and 1:4360, respectively. The photograph of the high-voltage generator is shown in Fig. 7. The generator has a small size, whose dimensions are $280 \mathrm{~mm} \times 150 \mathrm{~mm}$ in length and diameter, respectively.

\subsection{Measurement and results}

Commercially available software is used to calculate the charging voltage of $C_{2}$ of the circuit simulation schematic shown in Fig. 8. The values of circuit parameters in Fig. 8 are defined by the design values given in Table 1 . The simulation voltage waveform of charging $C_{2}$ is shown in Fig. 9. The waveform has a peak voltage of $460 \mathrm{kV}$ and a charging time of about $2.2 \mu$ s for reaching the peak in its first cycle.

In experiment, the breakdown voltage of the spark-gap

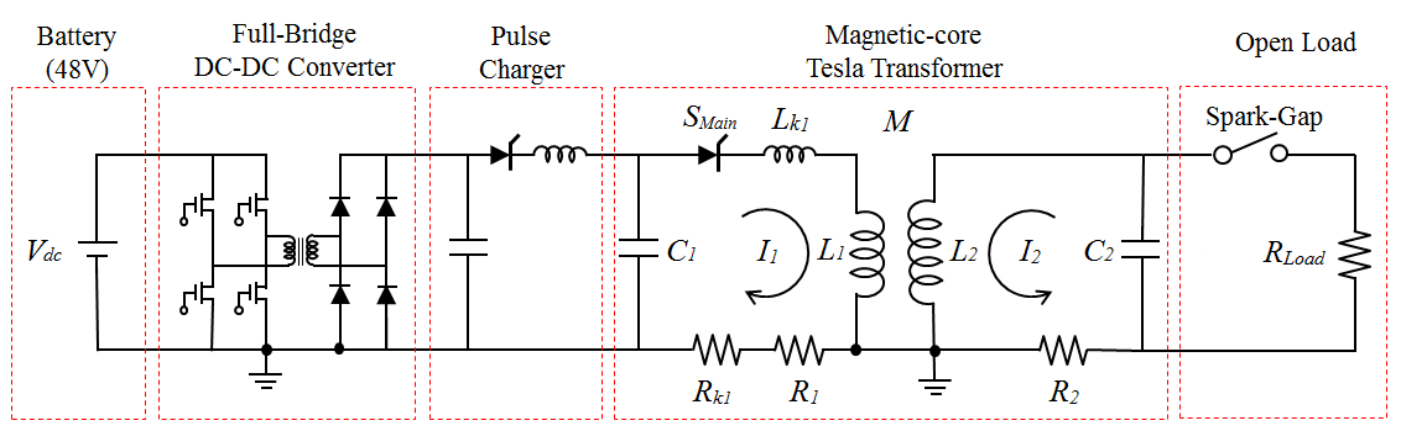

Fig. 5. Circuit diagram of the high-voltage generator

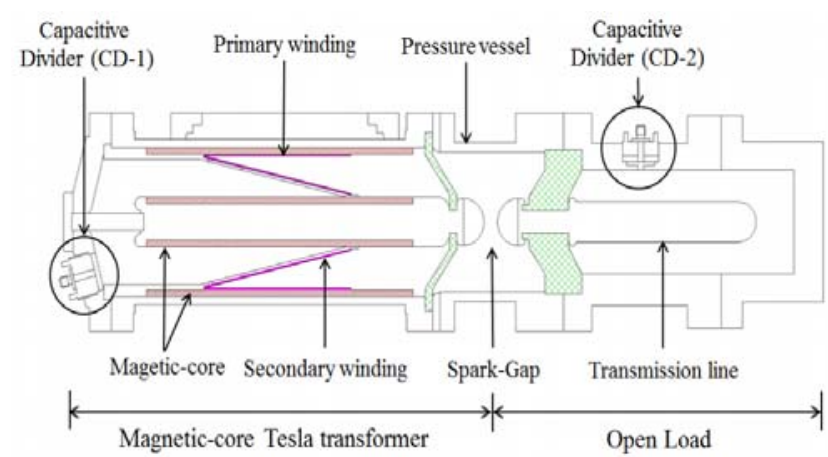

Fig. 6. Cross section diagram of the high-voltage generator.

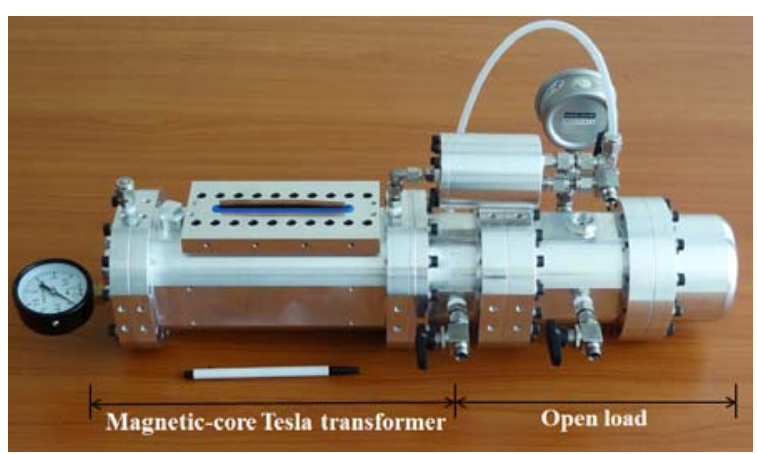

Fig. 7. Photograph of the high-voltage generator. 


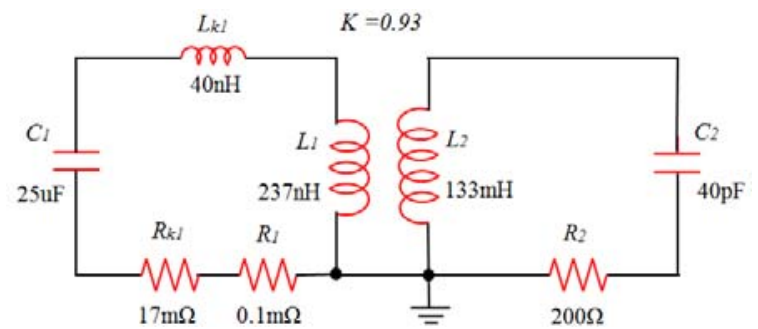

Fig. 8. Circuit simulation schematic of the transformer

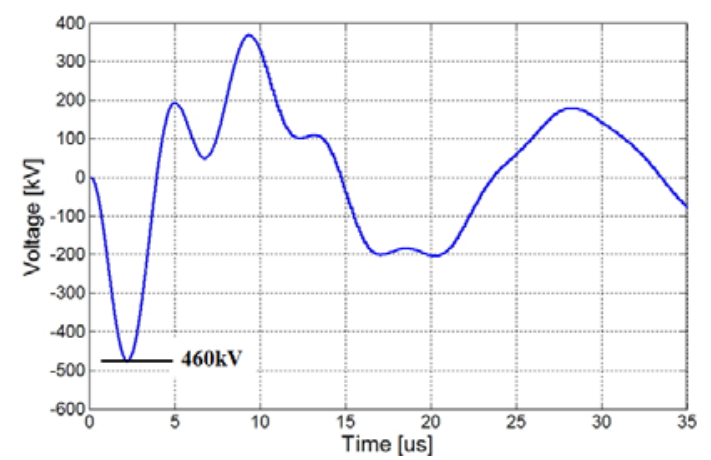

Fig. 9. Simulated voltage waveform of charging the secondary capacitor

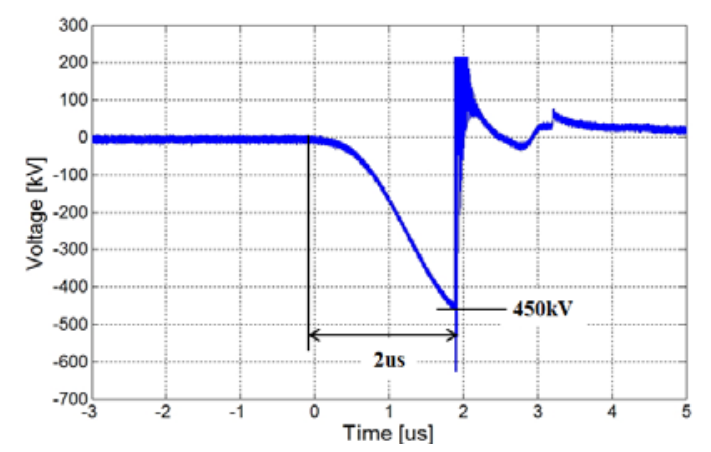

Fig. 10. Experiment voltage waveform of charging the secondary capacitor

switch is set to slightly lower voltage than the maximum charging voltage of the secondary capacitor. The experimental voltage waveform of charging $C_{2}$ is shown in Fig. 10.

When initial charging voltage of $C_{1}$ is $800 \mathrm{~V}$, the peaking charging voltage of $C_{2}$ is obtained as high as 450 $\mathrm{kV}$ and the charging time is about $2 \mu \mathrm{s}$. Experiment result shows good agreement with simulation one shown in Fig. 9. From the experiment result, the voltage gain of the transformer is $1: 563$ and energy transmission efficiency is calculated to be about $50 \%$.

A high-voltage pulse is generated when the transformer works together with spark-gap switch. Output voltage pulse of the high-voltage generator is shown in Fig. 11. It is clearly shown that the voltage pulse across the open load has an amplitude of $450 \mathrm{kV}$, a full width at half maximum (FWHM) of $2.5 \mathrm{~ns}$, and a rise time of $1.5 \mathrm{~ns}$. The design

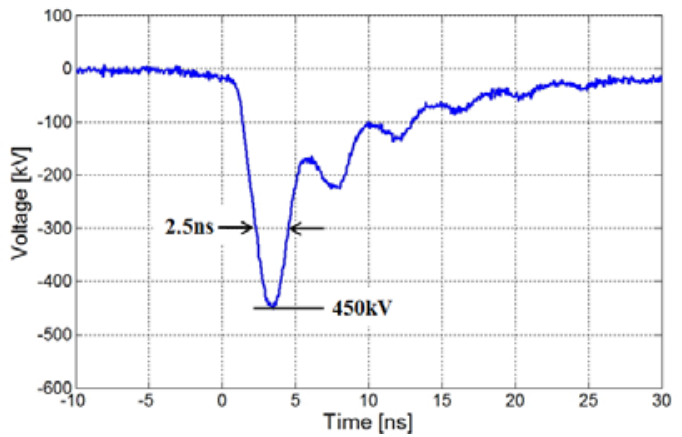

Fig. 11. Output voltage pulse of the high-voltage generator

value of FWHM is $2 \mathrm{~ns}$ by the Eq. (12). But, the measured value of FWHM is slightly increase by finite transition time of spark-gap switch. Output power of the high-voltage generator is calculated to be about $1.25 \mathrm{GW}$ for the $50 \Omega$ load. In the Fig. 11, there exist sequent step-like oscillations in the back edge of the waveform, which is result from the multiple voltage reflection due to the impedance mismatch between load and coaxial-type secondary capacitor.

\section{Conclusions}

A novel open-loop cylindrical magnetic-core applicable in the magnet-core Tesla transformer is proposed and manufactured. High-voltage generator based on Tesla transformer using the proposed magnetic-core is designed. The designed generator has a battery-powered operation and compact size of $280 \times 150 \mathrm{~mm}$ in length and diameter, respectively. Experiment results show that the generator can export high-voltage pulse with an amplitude of $450 \mathrm{kV}$ and FWHM of $2.5 \mathrm{~ns}$, with an energy conversion efficiency of about $50 \%$. Output power of the generator is about $1.2 \mathrm{GW}$ for the $50 \Omega$ load. The developed high-voltage generator can be applied for the compact and portable UWB HPEM source.

\section{References}

[1] F. Sabath, D. Nitsch, M. Jung, Th. H. G. G. Weise, "Design and Setup of a Short Pulse Simulator for Susceptibility Investigations, IEEE Transaction on plasma science, vol. 30, no. 5, pp. 1722-1727, Oct. 2002.

[2] L. Pecastaing, A. Silvestre de Ferron, M. Rivaletto, "Compact Self-loaded Marx Generator with Integrated Pulse-forming line for an Ultra-wideband source", Journal of the Korean Physical Society, vol. 59, no. 6, pp. 3463-3467, Dec. 2011.

[3] Y. Zhang, J. L. Liu, X. B. Cheng, G. Q. Bai, H. B. Zhang, J. H. Feng, and B. Liang, "A Compact Highvoltage Pulse Generator based on Pulse Transformer with Closed Magnetic Core", Review of Scientific 
Instruments. vol. 81, pp. 033302, 2010.

[4] Korovin, S. D., "Tesla Transformer in a High-current Repetitive Accelerator.", HCEI SB AN SSSR. Technical Report 47, pp.4-35, 1988.

[5] G. A. Mesyats, S. D. Korovin, V. V. Rostov, V. G. Shpak, M. I. Yalandin, "The RADAN series of Compact Pulsed Power Generators and Their Applications", Proceeding of the IEEE, vol. 92, no. 7, pp. 1166-1179, Jul. 2004.

[6] Bin-xiong Yu, Jin-liang Liu, "Parametric Calculation of Pulse Transformer with Open Magnetic Cores based on Magnetostatic-field Theory", Physical Review Special Topics-Accelerators and Beams, vol. 16, pp. 010401, 2013.

[7] Liu, J. L., Zhan, T. W., Zhang, J., Liu, Z. X., Feng, J. H., Shu, T., Zhang, J. D., Wang, X. X., “A Tesla pulse transformer for spiral water pulse forming line charging.", Laser and Particle Beams, vol. 25, issue 02, pp. 305-312, Jun. 2007.

[8] Jian-Chang Peng, Guo-Zhi Liu, Xiao-Xin Song, Jian-Cang $\mathrm{Su}$, "A high repetitive rate intense electron beam accelerator based on high coupling Tesla transformer.", Laser and Particle Beams, vol. 29, issue 01, pp. 55-60, Mar. 2011.

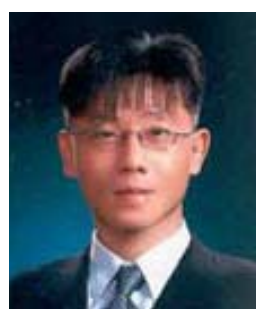

Young-Kyung Jeong received his M.S. degree in Computer Science \& Engineering, Changwon, Koera, in 1999. $\mathrm{He}$ is currently working on his Ph.D. course in the Department of Electrical and Computer Engineering, University of Seoul, Korea and a researcher-incharge in the Replex. Co., Ltd., Seoul, Korea. His research fields of interests are the HPEM and pulsed power systems.

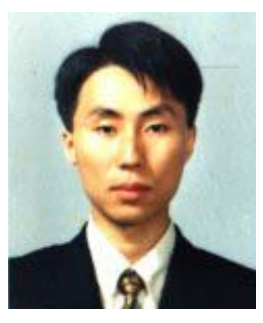

Dong-Gi Youn received M.S. and Ph.D. degrees in Electronics Engineering from Kyungnam University, Changwon, Korea, in 1997 and 2000, respectively. $\mathrm{He}$ is currently a chief executive officer in the Replex. Co., Ltd., Seoul, Korea. His research fields of interests are the HPEM and RF engineering.

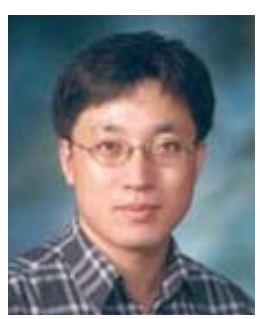

Moon-Que Lee received M.S. and Ph.D. degrees in Electronics Engineering from Seoul National University, Seoul, Korea, in 1994 and 1999, respectively. $\mathrm{He}$ is currently a Professor in the Department of Electrical and Computer Engineering, University of Seoul, Seoul, Korea. His research fields of interests are the design of RF-CMOS and MMIC. 